\title{
Light shifts in atomic Bragg diffraction
}

\author{
E. Giese ${ }^{1,2}$ A. Friedrich, ${ }^{2}$ S. Abend, ${ }^{3}$ E. M. Rasel,${ }^{3}$ and W. P. Schleich ${ }^{2,4}$ \\ (Published in Physical Review A 94, 063619 [2016].) \\ ${ }^{1}$ Department of Physics, University of Ottawa, 25 Templeton Street, Ottawa, ON, K1N 6N5, Canada. \\ ${ }^{2}$ Institut für Quantenphysik and Center for Integrated Quantum Science and Technology $\left(I Q^{S T}\right)$, \\ Universität Ulm, Albert-Einstein-Allee 11, D-89081, Germany. \\ ${ }^{3}$ Institut für Quantenoptik, Leibniz Universität Hannover, Welfengarten 1, D-30167 Hannover, Germany. \\ ${ }^{4}$ Texas A\&M University Institute for Advanced Study (TIAS), \\ Institute for Quantum Science and Engineering (IQSE) and Department of Physics and Astronomy, \\ Texas A\&M University, College Station, TX 77843-4242, USA.
}

\begin{abstract}
Bragg diffraction of an atomic wave packet in a retroreflective geometry with two counterpropagating optical lattices exhibits a light shift induced phase. We show that the temporal shape of the light pulse determines the behavior of this phase shift: In contrast to Raman diffraction, Bragg diffraction with Gaussian pulses leads to a significant suppression of the intrinsic phase shift due to a scaling with the third power of the inverse Doppler frequency. However, for box-shaped laser pulses, the corresponding shift is twice as large as for Raman diffraction. Our results are based on approximate, but analytical expressions as well as a numerical integration of the corresponding Schrdinger equation.
\end{abstract}

In the realm of high-precision measurements, the success of any method depends intimately on the suppression of uncertainties intrinsic to the technique. For this reason the phase shift caused by the light pulses forming an atom interferometer [1-4] is crucial, especially in view of recent ambitious projects as discussed in Refs. [5, 6]. In this article we focus on atomic Bragg diffraction, derive approximate, but analytical expressions for the twophoton light shift, and show that Gaussian light pulses lead to a significant suppression of this effect. Moreover, our analysis demonstrates that a naive translation of the results for Raman to Bragg diffraction might lead to a serious over- and underestimation of the light shift, depending on the pulse shape.

Nowadays, interferometers routinely employ a retroreflective mirror system with two running lattices to reduce the influence of wave-front distortions and vibrations [7]. The off-resonant pair of lasers in such a retroreflective setup [8] causes a light shift which translates into a phase shift which in turn depends on the initial atomic velocity. This so-called two-photon light shift is of particular relevance for any ambitious high-precision measurement employing retroreflected light fields as beam splitters and the correct incorporation of the phase shift is mandatory. Whereas this quantity has been thoroughly studied in Raman diffraction 9-11, we are not aware of a corresponding analysis for Bragg scattering [12, 13, the other major diffraction method for atoms [14. To constitute a competitive alternative to Raman, the light shifts in Bragg diffraction have to be controlled on a similar level of accuracy, especially since there is renewed interest [15-21] in this scattering mechanism and current setups 22 24] employ a retroreflective configuration.

In this article we (i) derive approximate, but analytical expressions for the two-photon light shift and compare them to the ones for Raman diffraction, (ii) confirm these results by numerically integrating the corresponding Schrdinger equation, and (iii) study the influence of the temporal shape of the pulses. Our approach not only enables us to accurately incorporate the phase contributions associated with the light pulses in the error budget, which so far was only possible for Raman diffraction, but also allows us to identify parameter regimes where, in comparison to Raman, the effects can be strongly suppressed.

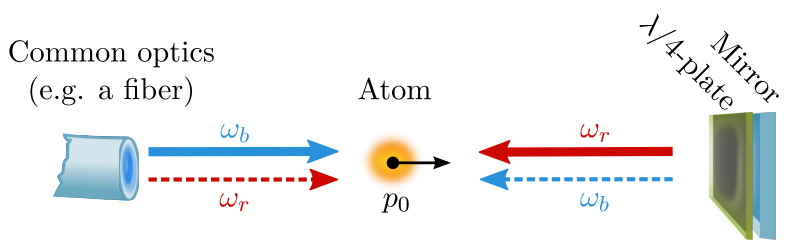

Figure 1. (Color online) Retroreflective setup for atomic Bragg diffraction. Two light fields of frequency $\omega_{b}$ and $\omega_{r}$ (light blue and red) with orthogonal polarization are guided by common optical elements, e.g., an optical fiber, to the atom. The polarization is changed by a quarter-wave-plate when the beams are reflected at the opposite side, leading to two distinguishable pairs of lasers represented by dashed and solid arrows. The degeneracy of the interaction between the two laser fields and the atom is lifted by an initial nonvanishing momentum $p_{0}$ of the atom, thus preventing double Bragg diffraction 25].

Figure 1 shows the corresponding setup where two light fields of frequencies $\omega_{b}$ and $\omega_{r}$ with orthogonal polarization are guided by common optical elements, e.g., an optical fiber, to an atomic sample and retroreflected on the opposite side. Since the polarization of the reflected laser beams is changed, the atom interacts effectively with two distinguishable [26] pairs of lasers, as indicated in Fig. 1 by solid and dashed lines. For an atom at rest the setup depicted in Fig. 1 is equivalent to the experimental configuration for double Bragg diffraction 
25, 27 30. However, if the atom has a non-vanishing initial momentum $p_{0}$, one laser pair can be chosen to induce the scattering process by adjusting the frequency difference $\Delta \omega=\omega_{b}-\omega_{r}$ leading to effective single Bragg diffraction in the retroreflective setup.

The phenomenon of Bragg scattering itself is a consequence of energy-momentum conservation, as illustrated by Fig. 2 We start from an atom which is initially in the ground state $|g\rangle$ with momentum $p_{0}$ and discuss the interaction with the pair of lasers represented in Fig. 1 by solid lines.

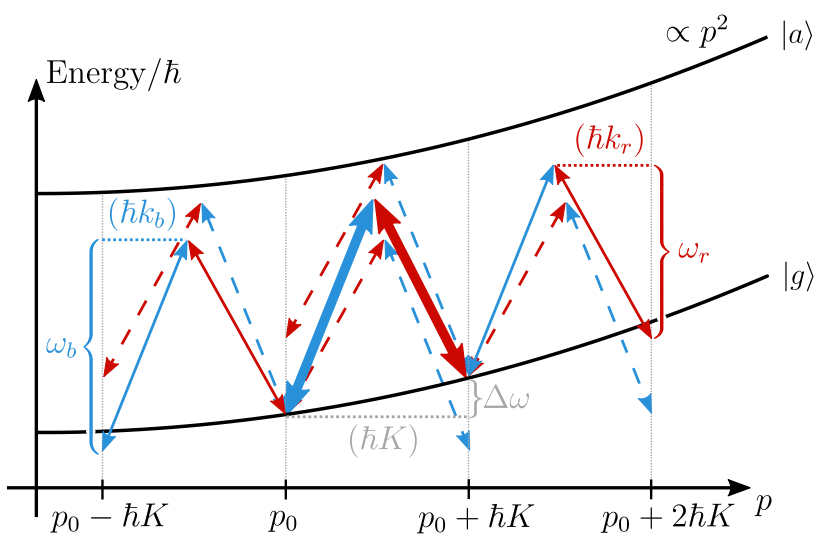

Figure 2. (Color online) Changes of energy (kinetic and internal) and momentum during a Bragg scattering process. Due to the absorption from or emission into the four laser fields shown in Fig. 1 the light blue (red) lasers lead to a momentum transfer of $\pm \hbar k_{b(r)}$ and an energy change of $\pm \hbar \omega_{b(r)}$, denoted by light blue (red) arrows. Because of energy and momentum conservation, the transition from $p_{0}$ to $p_{0}+\hbar K$ with $K=k_{b}+k_{r}$, induced by the solid pair of lasers in Fig. 1 is resonant (thick solid arrows). We draw the off-resonant transitions with thin lines and illustrate their deviation from the resonant energy. The dashed off-resonant transitions are induced by the second pair of lasers (depicted in Fig. 1 by dashed arrows).

First, the atom absorbs a photon of energy $\hbar \omega_{b}$ and is excited to an ancilla state $|a\rangle$. In this process it acquires the momentum $\hbar k_{b}$ of a photon. Since the transition is highly detuned from the ancilla state, the counterpropagating light field of energy $\hbar \omega_{r}$ stimulates the emission of a photon in the opposite direction, leading to an additional recoil of $\hbar k_{r}$. Thus, the total momentum transfer is $\hbar K \equiv \hbar k_{b}+\hbar k_{r}$, whereas the energy transferred by this two-photon process corresponds to the energy difference $\hbar \Delta \omega$. Hence, energy-momentum conservation is guaranteed for $\Delta \omega=\omega_{K}+\nu_{K}$, where we have defined the recoil and Doppler frequency by the expressions

$$
\omega_{K} \equiv \frac{\hbar K^{2}}{2 M} \quad \text { and } \quad \nu_{K}\left(p_{0}\right) \equiv \frac{p_{0} K}{M},
$$

respectively. Here, $M$ denotes the mass of the atom.

We emphasize that in the remainder of this article every expression involving the Doppler frequency $\nu_{K}\left(p_{0}\right)$ has an implicit dependence on the initial momentum $p_{0}$. However, we shall suppress this dependency for brevity of notation.

The previously described resonant process is illustrated in Fig. 2 by thick solid lines. As indicated by the thin solid lines, diffraction into the momenta $p_{0}-\hbar K$ and $p_{0}+2 \hbar K$ as well as higher orders is also possible. However, transitions to these momentum states violate energy conservation. Nevertheless, they may lead to energy shifts of the involved resonant states even if the population of the higher momentum states can be neglected. Since these shifts are symmetric for one pair of lasers this effect cancels out and a phase contribution introduced by the two-photon light shift cannot be observed for Bragg diffraction when performed without retroreflection.

However, the situation changes drastically when we consider the additional spurious pair of light fields (dashed arrows) in the retroreflective setup of Fig. 1. Here, the two colors of the light fields are exchanged, creating a whole variety of additional transitions illustrated in Fig. 2 by the thin dashed lines. Because all of these transitions are off-resonant, they may lead to a shift of the relevant energy levels and thus induce a phase difference between the two momentum states. In the remainder of this article we discuss this phase shift for $\pi / 2$ pulses.

In Raman scattering the large energy difference between the two relevant ground states allows us to neglect some of the off-resonant transitions [31] in the spirit of a rotating-wave approximation 32 .

Therefore, a perturbative treatment of the interaction of the atom with the laser fields leads [8, 9] us to the expression

$$
\delta \phi_{ \pm}^{(\mathrm{R})} \cong \pm \frac{\Omega}{4 \nu_{K}} \frac{\omega_{K} \pm \nu_{K}}{2 \omega_{K} \pm \nu_{K}}
$$

for the light shift for Raman diffraction. Here, $\Omega$ denotes the effective two-photon Rabi frequency, which depends on the intensity of the laser beams and the detuning from the ancilla state.

The different signs reflect the fact that the resonance condition of the scattering process can be adjusted such that the atom is scattered either towards the retroreflective mirror or away from it. In fact, while we have chosen $\Delta \omega=\omega_{K}+\nu_{K}$ during the previous discussion leading to a resonant coupling between the momenta $p_{0}$ and $p_{0}+\hbar K$, we can alternatively choose $\Delta \omega=\omega_{K}-\nu_{K}$ which corresponds to diffraction into the opposite direction, i. e., a coupling of the momenta $p_{0}$ and $p_{0}-\hbar K$. In Raman diffraction the analog feature is usually employed [33, 34] to compensate for systematic errors.

Since in Bragg diffraction the internal state of the atom is not changed, more off-resonant transitions have to be taken into account, which makes a perturbative treatment more challenging. If we describe an atom in the ground state and momentum eigenstate $\left|p_{0}+n \hbar K\right\rangle$ by the probability amplitude $g_{n}$, an adiabatic elimination of 
the ancilla state leads 28] to the system 35]

$$
\begin{aligned}
\dot{g}_{n} & =i \frac{\Omega}{2}\left(\mathrm{e}^{-i \theta} \mathrm{e}^{2 i\left(n \omega_{K}+\nu_{K}\right) t}+\mathrm{e}^{i \theta} \mathrm{e}^{2 i(n-1) \omega_{K} t}\right) g_{n-1} \\
& +i \frac{\Omega}{2}\left(\mathrm{e}^{i \theta} \mathrm{e}^{-2 i\left[(n+1) \omega_{K}+\nu_{K}\right] t}+\mathrm{e}^{-i \theta} \mathrm{e}^{-2 i n \omega_{K} t}\right) g_{n+1}
\end{aligned}
$$

of differential equations describing the diffraction process. Here, $\theta$ denotes the phase difference of the two counterpropagating laser fields.

The phase caused by the light shift can be found by solving Eq. (3) and determining the phase of the complexvalued ratio $g_{1} / g_{0}$ for $\Omega t=\pi / 2$. We therefore apply, in complete analogy to Ref. [36], the resonance conditions $\Delta \omega=\omega_{K} \pm \nu_{K}$ and perform the method of averaging [37. We then calculate the argument of $g_{1} / g_{0}$ for $\Omega t=\pi / 2$ in a similar manner as in Ref. [38 resulting in the approximate expression

$$
\delta \phi_{ \pm}^{(\mathrm{B})} \cong \frac{\Omega}{4} \frac{2}{\omega_{K} \pm \nu_{K}} \pm \frac{\Omega}{4 \nu_{K}} \frac{\omega_{K}^{2}}{\left(2 \omega_{K} \pm \nu_{K}\right)\left(\omega_{K} \pm \nu_{K}\right)}
$$

for the phase induced by light shifts in Bragg diffraction with box-shaped laser pulses.

Strictly speaking, Eq. (4) is only valid for $\nu_{K}$ being an integer multiple of $\omega_{K}$ since only then the timedependent coupling in Eq. (3) leads to a clear separation of two different frequency scales, as required by the method of averaging. However, when we compare our approximate result in Fig. 3 to a numerical solution of Eq. (3), we find that it is exact at integer values of $\nu_{K} / \omega_{K}$ and between them the relative deviation oscillates within $2 \%$.

Moreover this comparision shows that Eqs. (2) and (4) are only good approximations for sufficiently large $\nu_{K}$, since for vanishing initial momentum the diffraction process changes drastically to double diffraction, and hence our identification of the resonant momentum states is not appropriate anymore. Indeed, the poles in the analytic result, i.e. Eq. (4), as well as the deviation of the numerical simulation from the analytical results for the phase shift for small $\nu_{K} / \omega_{K}$, apparent in Fig. 3, are due to a modified diffraction mechanism. Each of the poles in Eq. (4) can be attributed to a specific case [36] of double or degenerate diffraction. Moreover, we note that an ana$\log$ analytical treatment can be performed [36] to obtain the light shift where these equations diverge.

Of particular interest for this article is the scaling behavior of the phase shift $\delta \phi_{ \pm}^{(B)}$ for a large Doppler detuning, that is, for $\omega_{K} / \nu_{K} \ll 1$. We note that the asymptotic behavior of Eq. (4), in lowest order, is determined entirely by the first term 39 providing us with the scaling

$$
\delta \phi_{ \pm}^{(\mathrm{B})} \cong \pm \frac{\Omega}{2 \nu_{K}} \cong 2 \delta \phi_{ \pm}^{(\mathrm{R})},
$$

which is twice the light-shift phase of Raman diffraction. The asymptotic behavior and the comparison to the Raman case are also illustrated by Fig. 3 .
Even though there are more relevant transitions in Bragg diffraction, the overall scaling of the light shift does not change significantly. This behavior is due to the intricate combination and cancelation effects [36] between the individual shifts of the energy levels due to the additional off-resonant transitions indicated in Fig. 2

In fact, in Fig. 2 the momentum $p_{0}$ is connected to $p_{0}+\hbar K$ not only by the solid resonant lasers, but also by the dashed Doppler-detuned pair of lasers. This interaction leads to a change of the population which makes a contribution to the phase induced by the light shift. Indeed, this population contribution is the origin of the first addend in Eq. (4).

Since this effect is suppressed for time-dependent adiabatic pulses 43, the level shifts induced by the various off-resonant transitions cancel out partially and the phase shift is dominated by the second term in Eq. (4). To acquire an intuition for adiabatic pulses we consider Gaussian pulses and neglect the first contribution to the phase shift in Eq. (4). Hence, we conjecture the expression

$$
\delta \phi_{ \pm}^{(\mathrm{ad})} \cong \Phi[\Omega] \frac{\omega_{K}^{3}}{ \pm \nu_{K}\left(2 \omega_{K} \pm \nu_{K}\right)\left(\omega_{K} \pm \nu_{K}\right)}
$$

for the phase induced by the two-photon light shift of an adiabatic Gaussian pulse. Here, we have introduced the dimensionless amplitude

$$
\Phi[\Omega(t)] \equiv \frac{\int \Omega^{2}(t) \mathrm{d} t}{4 \omega_{K} \int \Omega(t) \mathrm{d} t},
$$

and have replaced [40] the two-photon Rabi frequency $\Omega$ by its time-dependent analog $\Omega(t)$.

In order to verify our conjecture, we solve Eq. (3) numerically for a time-dependent Gaussian pulse and determine the phase of the complex-valued ratio $g_{1} / g_{0}$ for a pulse area of $\int \Omega(t) \mathrm{d} t=\pi / 2$. Figure 3 demonstrates that the light shift as predicted by our analytical expression Eq. (6) captures the main features of the numerical solution and can therefore serve as an estimate for the light shift with a Gaussian pulse. In particular, we observe a relative deviation in the order of $4 \%$, which decreases further with increasing initial momentum. Furthermore, by expanding Eq. (6) in lowest order for $\omega_{K} / \nu_{K} \ll 1$ we obtain the scaling law

$$
\delta \phi_{ \pm}^{(\mathrm{ad})} \cong \pm \Phi[\Omega]\left(\frac{\omega_{K}}{\nu_{K}}\right)^{3},
$$

which has a completely different scaling from that of Eq. (5), leading to a suppression of the phase shift with the third power of the inverse Doppler frequency. This behavior is a significant benefit for large initial momenta as demonstrated by Fig. 3 .

We are now in the position to summarize our key results, compare the magnitude of the two-photon light shift to the typical phase sensitivity of state-of-the-art measurements and provide an outlook for future work. 

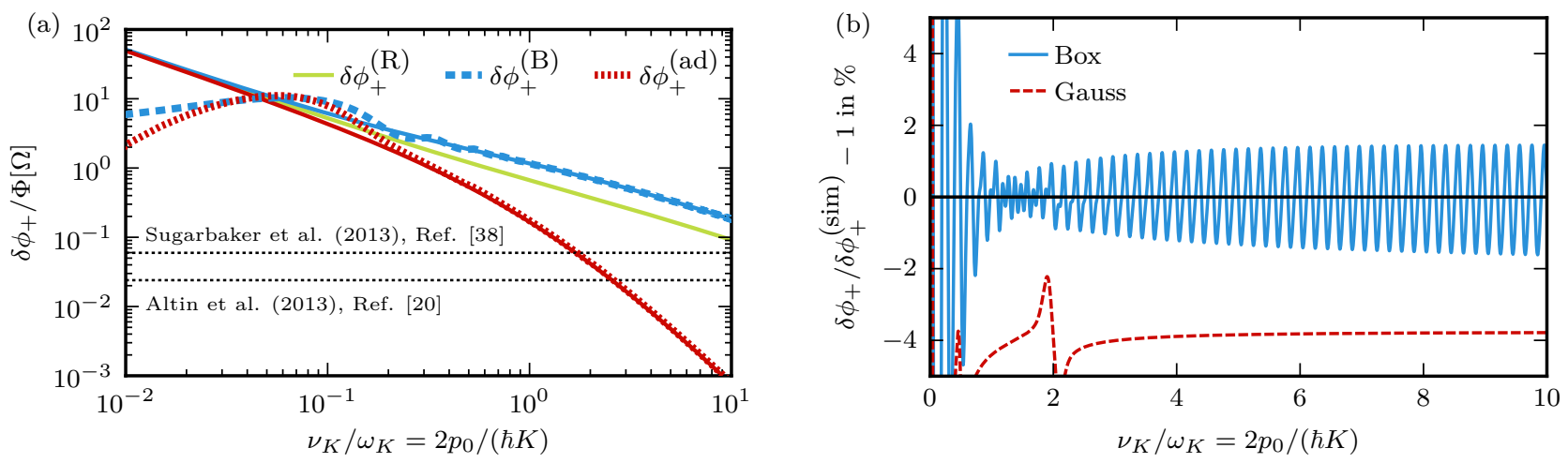

Figure 3. (Color online) Scaling of the two-photon light shift $\delta \phi_{+}$with respect to the initial momentum $p_{0}$ (a), and relative deviation of the analytic expressions from the numerical simulation (b). In panel (a) we exhibit three cases: (i) Raman diffraction [(R), solid green], (ii) Bragg diffraction with box-shaped pulses [(B), dashed blue], and (iii) Bragg diffraction with Gaussian pulses $\left[(\mathrm{ad})\right.$, dotted red]. We scale $\delta \phi_{+}$in terms of the amplitude $\Phi[\Omega]$ defined 40 by Eq. (7) and use Eq. (1) to obtain $\nu_{K} / \omega_{K}=2 p_{0} /(\hbar K)$. Whereas the light shift in Bragg with box-shaped pulses is twice the size of the Raman case, but scales identically with increasing Doppler detuning, it is largely suppressed for adiabatically tuned pulses. The solid lines represent the analytical solution; the dashed and dotted lines are numerical simulations, respectively. To illustrate the order of magnitude of the effect we compare the two-photon light shift to the phase uncertainty in two state-of-the-art experiments [22, 41, indicated by the horizontal dotted lines [42. Panel (b) shows that the numerical solution for box pulses [solid blue] oscillates within $2 \%$ of the corresponding analytical solution and coincides with it for integer values of $\nu_{K} / \omega_{K}$. For Gaussian pulses [dashed red] the analytical solution provides an estimate for the light shift and we observe an agreement with the theoretical prediction to within $4 \%$, which decreases further with higher initial momenta $p_{0}$. However, since the light shift for Gaussian pulses is highly suppressed for large initial momenta the absolute deviation is significantly smaller than in the case of box pulses.

For this purpose we first recall one more time the motivation of our study, which also underscores its relevance for experiments. For high-precision measurements with atom interferometers using Bragg diffraction the form and magnitude of the phase induced by the two-photon light shift are essential for both the error estimation as well as possible mitigation strategies, based on measurements performed with a reversed momentum transfer or appropriately chosen laser intensities [34. Therefore, the approximate but analytical expression provided in our article constitutes a vital part of the analysis of phase contributions in Bragg interferometers and is of relevance for any ambitious experiment employing this diffraction technique.

Indeed we have shown that for box-shaped laser pulses the phase induced by the two-photon light shift is roughly twice as large as in Raman diffraction. Apart from this factor of two, it is of the same form, and thus the methods 34] used to compensate for this shift can be directly applied.

Adiabatic pulse shapes are a convenient way in Bragg diffraction to prevent scattering into higher momentum states 25, 44 and thus are employed by most experiments. We have shown that by using such pulses the light shifts are suppressed, an effect that has no direct correspondence in Raman diffraction. In contrast for Bragg diffraction, the phase shift for adiabatic Gaussian laser pulses scales favorably with the third power of the inverse Doppler frequency. On top of the benefits from this new scaling behavior, our results suggest that the conventional mitigation strategies 34 can also be applied.

Our analysis shows that in a Mach-Zehnder interferometer with an initial momentum but no acceleration, the contributions of the first and final light pulse cancel exactly as the same phase is imprinted on both interferometer arms. However, in current gravimeters where atoms are released from a trap, the light shifts will not compensate each other due to the acceleration of the atomic wave packets between the pulses. Moreover, for small initial momenta, the light-shift contribution to the phase is dominated by the first pulse. Hence, with a delayed start of the interferometer sequence, the atoms can be accelerated and the superior scaling behavior of Bragg diffraction with Gaussian pulses can be exploited, leading to a smaller overall light shift.

In contrast to that, in a fountain experiment the light shifts caused by the beam splitters do not cancel due to the reversed momentum of the atom. A suppression of the phase shift then only results from the different scaling behavior and a large initial momentum, which can be applied to minimize the light shift phase 45.

When we compare our results to state-of-the-art experiments in Fig. 3(a), we see that the magnitude of the effect due to the different scaling behavior is less important than in Raman diffraction, but might not be negligible in all cases. The horizontal dotted lines show the phase uncertainty of Ref. 22] and Ref. 41] scaled with $\Phi[\Omega]$ performed with a retroreflective setup using Raman and Bragg diffraction, respectively. For every specific setup- 
especially in gravimetric applications - relevant parameters for the determination of the phase shift are the pulse shape, pulse duration, pulse sequence, interrogation time, acceleration, initial momentum, atomic species and more. Thus, the comparison provided above might only be seen as an rough estimate of the order of magnitude for the light shift. However, when designing new experiments, a more detailed analysis is called for which can be obtained straightforwardly for an individual setup from our results 42 .

Whereas in this article we have focused on phase shifts caused by the spurious pair of lasers, we investigate in Ref. 36] the diffraction in a retroreflective setup itself without solely focusing on light shifts. There, we not only derive analytical expressions for the diffraction incorporating the spurious pair of lasers (which can be used to obtain the expressions for the phase shift), but also perform extensive numerical studies of box-shaped Bragg pulses for different initial momenta. In particular, we discuss the transition to double Bragg diffraction for momenta where Eq. (4) diverges. Moreover, we employ different adiabatic pulse shapes to verify the analytical result, that is Eq. (6).

Our numerical analysis can easily be generalized to shed light on the two-photon light shift for a broad momentum distribution of the atom 46] or higher-order Bragg diffraction. Moreover, we admit that in a strict sense our analysis is only valid for perfectly orthogonal polarization. Since in an experiment, effects of imperfect polarization can be determined and, if possible, mini- mized [9] we plan to investigate the effect of polarization on the light shift.

The final judgment of any physical theory is experiment and high-precision measurements are a key ingredient in probing the foundations of physics. In this spirit we hope that an increased accuracy of Bragg interferometers made possible by our expressions, paves the road to novel applications and a verification of fundamental physical theories and concepts.

\section{ACKNOWLEDGMENTS}

We thank H. Ahlers, M. A. Efremov, S. Kleinert, P. Kling, V. S. Malinovsky, M. Meister, A. Roura, C. Schubert, V. Tamma, C. Ufrecht, and W. Zeller for many fruitful discussions. This project is supported by the German Space Agency (DLR) with funds provided by the Federal Ministry for Economic Affairs and Energy (BMWi) due to an enactment of the German Bundestag under Grant Numbers DLR 50WM1552-1557 (QUANTUS-IV-Fallturm). W.P.S. is grateful to Texas A\&M University for a Texas A\&M University Institute for Advanced Study (TIAS) Faculty Fellowship. E.G. and A.F. thank the Center for Integrated Quantum Science and Technology $\left(\mathrm{IQ}^{\mathrm{ST}}\right)$ for a fellowship. E.G. acknowledges the support of the Friedrich-AlexanderUniversität Erlangen-Nürnberg through an Eugen Lommel Stipend.
[1] Ch. J. Bord, Phys. Lett. A 140, 10 (1989).

[2] M. Kasevich and S. Chu, Phys. Rev. Lett. 67, 181 (1991)

[3] G. M. Tino and M. A. Kasevich, eds., Atom Interferometry, Proceedings of the International School of Physics "Enrico Fermi", Course 188, Italian Physical Society (IOS Press, Amsterdam, 2014).

[4] S. Kleinert, E. Kajari, A. Roura, and W. P. Schleich, Phys. Rep. 605, 1 (2015).

[5] L. Zhou, S. Long, B. Tang, X. Chen, F. Gao, W. Peng, W. Duan, J. Zhong, Z. Xiong, J. Wang, Y. Zhang, and M. Zhan, Phys. Rev. Lett. 115, 013004 (2015).

[6] J. M. Hogan and M. A. Kasevich, Phys. Rev. A 94, 033632 (2016)

[7] See for example A. Peters, K. Y. Chung, and S. Chu, Metrologia 38, 25 (2001).

[8] P. Cladé, E. de Mirandes, M. Cadoret, S. GuellatiKhélifa, C. Schwob, F. Nez, L. Julien, and F. Biraben, Phys. Rev. A 74, 052109 (2006).

[9] A. Gauguet, T. E. Mehlstäubler, T. Lévèque, J. Le Gouët, W. Chaibi, B. Canuel, A. Clairon, F. Pereira Dos Santos, and A. Landragin, Phys. Rev. A 78, 043615 (2008).

[10] O. Carraz, R. Charrière, M. Cadoret, N. Zahzam, Y. Bidel, and A. Bresson, Phys. Rev. A 86, 033605 (2012)

[11] P. Gillot, B. Cheng, S. Merlet, and F. Pereira Dos Santos, Phys. Rev. A 93, 013609 (2016).
[12] Other effects of off-resonant laser pairs in Bragg diffraction such as diffraction phases have been studied for example in B. Estey, C. Yu, H. Müller, P.-C. Kuan, and S.-Y. Lan, Phys. Rev. Lett. 115, 083002 (2015).

[13] Diffraction phases for an interferometer operated in the Raman-Nath regime have been discussed in M. Büchner, R. Delhuille, A. Miffre, C. Robilliard, J. Vigué, and C. Champenois, Phys. Rev. A 68, 013607 (2003).

[14] M. Kozuma, L. Deng, E. W. Hagley, J. Wen, R. Lutwak, K. Helmerson, S. L. Rolston, and W. D. Phillips, Phys. Rev. Lett. 82, 871 (1999),

[15] H. Müller, S.-w. Chiow, Q. Long, S. Herrmann, and S. Chu, Phys. Rev. Lett. 100, 180405 (2008).

[16] P. Cladé, T. Plisson, S. Guellati-Khélifa, F. Nez, and F. Biraben, Eur. Phys. J. D 59, 349 (2010)

[17] S.-w. Chiow, T. Kovachy, H.-C. Chien, and M. A. Kasevich, Phys. Rev. Lett. 107, 130403 (2011).

[18] J. E. Debs, P. A. Altin, T. H. Barter, D. Döring, G. R. Dennis, G. McDonald, R. P. Anderson, J. D. Close, and N. P. Robins, Phys. Rev. A 84, 033610 (2011).

[19] T. Kovachy, S.-w. Chiow, and M. A. Kasevich, Phys. Rev. A 86, 011606 (2012).

[20] B. Canuel, L. Amand, A. Bertoldi, W. Chaibi, R. Geiger, J. Gillot, A. Landragin, M. Merzougui, I. Riou, S. Schmid, and P. Bouyer, E3S Web Conf. 4, 01004 (2014).

[21] J. Hartwig, S. Abend, C. Schubert, D. Schlippert, 
H. Ahlers, K. Posso-Trujillo, N. Gaaloul, W. Ertmer, and E. M. Rasel, New J. Phys. 17, 035011 (2015).

[22] P. A. Altin, M. T. Johnsson, V. Negnevitsky, G. R. Dennis, R. P. Anderson, J. E. Debs, S. S. Szigeti, K. S. Hardman, S. Bennetts, G. D. McDonald, L. D. Turner, J. D. Close, and N. P. Robins, New J. Phys. 15, 023009 (2013).

[23] T. Kovachy, P. Asenbaum, C. Overstreet, C. A. Donnelly, S. M. Dickerson, A. Sugarbaker, J. M. Hogan, and M. A. Kasevich, Nature 528, 530 (2015).

[24] G. D'Amico, F. Borselli, L. Cacciapuoti, M. Prevedelli, G. Rosi, F. Sorrentino, and G. M. Tino, Phys. Rev. A 93, $063628(2016)$.

[25] E. Giese, A. Roura, G. Tackmann, E. M. Rasel, and W. P. Schleich, Phys. Rev. A 88, 053608 (2013)

[26] Reference 9] makes a similar assumption in the analytical treatment of Raman diffraction and studies the effects of standing waves experimentally.

[27] T. Lévèque, A. Gauguet, F. Michaud, F. Pereira Dos Santos, and A. Landragin, Phys. Rev. Lett. 103, 080405 (2009)

[28] E. Giese, Fortschr. Phys. 63, 337 (2015)

[29] H. Ahlers, H. Müntinga, A. Wenzlawski, M. Krutzik, G. Tackmann, S. Abend, N. Gaaloul, E. Giese, A. Roura, R. Kuhl, C. Lämmerzahl, A. Peters, P. Windpassinger, K. Sengstock, W. P. Schleich, W. Ertmer, and E. M. Rasel, Phys. Rev. Lett. 116, 173601 (2016).

[30] J. Küber, F. Schmaltz, and G. Birkl, arXiv:1603.08826 [cond-mat.quant-gas] (2016).

[31] In fact, in a retroreflective setup, energy of this order is added to and subtracted from the respective energy of the internal state. However, for an atom in the lower state the transition energy associated with the subtraction is far off-resonant and usually neglected. The same is true for the addition of the energy when the atom is in the upper state.

[32] W. Schleich, Quantum Optics in Phase Space (WileyVCH, Weinheim, 2001).

[33] J. M. McGuirk, G. T. Foster, J. B. Fixler, M. J. Snadden, and M. A. Kasevich, Phys. Rev. A 65, 033608 (2002)

[34] A. Louchet-Chauvet, T. Farah, Q. Bodart, A. Clairon, A. Landragin, S. Merlet, and F. Pereira Dos Santos, New J. Phys. 13, 065025 (2011).

[35] Equation (3) emerges by setting $\Delta \omega=\omega_{K}+\nu_{K}$ in the derivation of the differential equations 25] leading to double Bragg diffraction. A similar equation for diffrac- tion in the opposite direction originates from the choice $\Delta \omega=\omega_{K}-\nu_{K}$.

[36] A. Friedrich, E. Giese, E. M. Rasel, and W. P. Schleich, "Impact of retroreflection on atomic Bragg diffraction," (2016), in preparation.

[37] N. N. Bogoliubov and Y. A. Mitropolsky, Asymptotic methods in the theory of non-linear oscillations (Hindustan Publishing Corpn., Delhi, 1961).

[38] D. S. Weiss, B. C. Young, and S. Chu, Appl. Phys. B 59, 217 (1994).

[39] Indeed, this fact stands out most clearly, when we cast the first term in Eq. (4) into the form $\Omega /\left[4\left(\omega_{K} \pm \nu_{K}\right)\right]=$ $2 \delta \phi^{(\mathrm{R})}\left\{1-\left[\omega_{K} /\left(\omega_{K} \pm \nu_{K}\right)\right]^{2}\right\}$, which connects the phase shift in Raman diffraction, i.e. Eq. (2), to the one induced by the light shift in Bragg diffraction even beyond the asymptotic regime.

[40] The integral over $\Omega^{2}(t)$ occurs since the two-photon light shift is a higher-order process. Furthermore, the scaling factor $\Phi[\Omega]$ reduces to the factor that appears in Eqs. (2), (4), and (6) for box-shaped pulses, since for a pulse duration of $t$ we have $\Phi[\Omega]=\Omega^{2} t /\left(4 \omega_{K} \Omega t\right)=$ $\Omega /\left(4 \omega_{K}\right)$. In the case of a time-dependent Gaussian pulse $\Omega(t)$ with maximal amplitude $\Omega$ we find $\Phi[\Omega(t)]=$ $\Omega /\left(4 \sqrt{2} \omega_{K}\right)$.

[41] A. Sugarbaker, S. M. Dickerson, J. M. Hogan, D. M. S. Johnson, and M. A. Kasevich, Phys. Rev. Lett. 111, 113002 (2013)

[42] For the comparison we use the definition of a Gaussian pulse $\Omega(t) \equiv \Omega_{0} \exp \left[-t^{2} /\left(2 \sigma^{2}\right)\right]$ and $\Phi[\Omega] \equiv$ $(16 / \sqrt{\pi}) \omega_{K} \sigma$. The recoil frequency of Rubidium atoms is $\omega_{K}=15 \cdot 2 \pi \mathrm{kHz}$. In case of Ref. [22] we find a phase uncertainty of $1.5 \mathrm{mrad}$ and $\sigma=15 \mu \mathrm{s}$. Even tough the specific pulse shape is not stated in Ref. [41, we suspect that it is also Gaussian and therefore we use $\sigma=35 \mu \mathrm{s}$ and a phase uncertainty of $2 \mathrm{mrad}$.

[43] V. S. Malinovsky and P. R. Berman, Phys. Rev. A 68, $023610(2003)$

[44] H. Müller, S.-w. Chiow, and S. Chu, Phys. Rev. A 77, 023609 (2008)

[45] S. Abend, M. Gebbe, M. Gersemann, H. Ahlers, H. Müntinga, E. Giese, N. Gaaloul, C. Schubert, C. Lämmerzahl, W. Ertmer, W. P. Schleich, and E. M. Rasel, Phys. Rev. Lett. 117, 203003 (2016).

[46] S. S. Szigeti, J. E. Debs, J. J. Hope, N. P. Robins, and J. D. Close, New J. Phys. 14, 023009 (2012) 